\title{
(2) OPEN ACCESS \\ Impact of comorbidities on clinical prognosis in 1280 patients with different types of COVID-19
}

\author{
Huilin Fang, Qiaomei Liu, Maomao Xi, Di Xiong, Jing He, Pengcheng Luo, \\ Zhanghua Li
}

Additional material is published online only. To view, please visit the journal online (http://dx.doi.org/10. 1136/jim-2020-001555)

Wuhan Third Hospital Tongren Hospital of Wuhan University, Wuhan, China

\section{Correspondence to}

Dr Zhanghua Li, Wuhan Third Hospital, Tongren Hospital of Wuhan University, Wuhan 430074, China;

Izh999999@aliyun.com

Accepted 25 September 2020

Published Online First

13 October 2020

\section{Check for updates}

(C) American Federation for Medical Research 2021 Re-use permitted under CC BY-NC. No commercial re-use. Published by BMJ.

To cite: Fang $\mathrm{H}$, Liu Q, Xi M, et al. J Investig Med 2021;69:75-85.

\section{ABSTRACT}

The study aimed to compare the clinical characteristics and outcomes of patients with different types (ordinary, severe, and critical) of COVID-19. A total of 1280 patients diagnosed with COVID-19 were retrospectively studied, including 793 ordinary patients, 363 severe patients and 124 critical patients. The impact of comorbidities on prognosis in ordinary, severe, and critical patients were compared and analyzed. The most common comorbidities were hypertension (33.0\%), followed by diabetes (14.4\%). The length of hospital stay and time from the onset to discharge were significantly longer in ordinary patients with comorbidities compared with those without comorbidities. Critical patients with comorbidities had significantly lower cure rate ( $19.3 \%$ vs $38.9 \%, p<0.05)$ and significantly higher mortality rate ( $53.4 \%$ vs $33.3 \%$, $\mathrm{p}<0.05)$ compared with those without comorbidities. The time from onset to discharge was significantly longer in ordinary patients with hypertension compared with those without hypertension. The mortality rate of critical patients with diabetes was higher than that of patients without diabetes $(71.4 \%$ vs $42.7 \%$, $\mathrm{p}<0.05)$. Men had a significantly increased risk of death than women (OR=4.395, 95\% Cl 1.896 to 10.185 , $p<0.05)$; patients with diabetes had higher risk of death $(\mathrm{OR}=3.542,95 \% \mathrm{Cl} 1.167$ to $10.750, p<0.05)$. Comorbidities prolonged treatment time in ordinary patients, increased the mortality rate and reduced the cure rate of critical patients; hypertension and diabetes may be important factors affecting the clinical course and prognosis of ordinary and critical patients, respectively.

\section{INTRODUCTION}

Since December 2019, cases with unexplained pneumonia have been reported in many countries throughout the world. The disease is mainly manifested by fever, dry cough and fatigue. ${ }^{1}$ On January 7, 2020, Chinese research successfully isolated new coronavirus strains from the respiratory specimens of infected patients. On January 12, 2020, the WHO named the new coronavirus causing pneumonia epidemic as 2019 new coronavirus or 2019nCoV. On February 11, 2020, the International Committee on Taxonomy of Viruses renamed the virus as SARS-CoV-2. Subsequently, the WHO officially named the disease caused by SARS-CoV-2 as COVID-19. ${ }^{2-4}$ The population

\section{Significance of this study}

What is already known about this subject?

- COVID-19 is a new type of respiratory infectious disease caused by SARS-CoV-2.

- Previous studies have shown that comorbidities are a risk factor for severity and mortality in patients with COVID-19.

- However, the specific impact of comorbidities on patients with different types of COVID-19 has been rarely reported.

What are the new findings?

- This study, to our knowledge, is the first study that investigates the impact of comorbidities on prognosis in patients with three different types (ordinary, severe, and critical) of COVID-19.

- Comorbidities did not affect the cure rate and the mortality rate of ordinary patients, but can prolong patients' clinical course, including length of hospital stay and time to cure.

- Comorbidities have no obvious impact on the cure rate, mortality rate and clinical course of severe patients.

- Comorbidities increased the mortality rate, reduced the cure rate, and prolonged the clinical course of critical patients.

- Hypertension may be an important factor affecting the clinical course of ordinary patients, and diabetes may be an important factor affecting the clinical course and prognosis of critical patients.

How might these results change the focus of research or clinical practice?

- Comorbidities, especially hypertension and diabetes are associated with poorer clinical outcomes in ordinary and critical patients. Evaluation of comorbidity is of importance in patients with COVID-19.

is generally susceptible to COVID-19, which is a highly contagious infectious disease, and spreads rapidly around the world. The WHO has declared the outbreak of COVID-19 to be a public health emergency of international concern. ${ }^{5-7}$ 
To date, many studies have reported the epidemiological and clinical features of COVID-19. The clinical features of COVID-19 are varied, including asymptomatic infection, mild upper respiratory tract infection, severe viral pneumonia complicated by respiratory failure, and even death. The most common clinical symptoms include fever, dry cough, fatigue, sputum production, dyspnea, sore throat, myalgia, diarrhea, and headache. Some patients with COVID-19 can rapidly progress to acute respiratory distress syndrome. ${ }^{128}$ A meta-analysis showed that about $52 \%$ of patients have been cured and discharged after treatment, but $5 \%$ of patients had died. ${ }^{9}$ Multiple studies have shown that advanced age ( $>65$ years), male sex, and comorbidities are independent risk factors for disease severity and death from COVID-19. ${ }^{10-12}$ Other studies showed that 20\%-51\% of patients with COVID-19 have at least one comorbidity, and the most common comorbidities are hypertension (21.1\%) and diabetes (9.7\%), followed by cardiovascular disease (8.4\%) and respiratory diseases $(1.5 \%) .{ }^{1213} \mathrm{~A}$ recent study showed that hypertension, diabetes, chronic obstructive pulmonary disease (COPD), and malignant tumors are risk factors for severity (intensive care unit (ICU) transfer and endotracheal intubation) and death in patients with COVID-19. ${ }^{14}$ Although previous studies have shown that comorbidities are a risk factor for severity and mortality in patients with COVID-19, the specific impact of comorbidities on patients with different types of COVID-19 has been rarely reported. In this study, we included patients with COVID-19, divided them into ordinary, severe, and critical types according to the clinical manifestations at admission, and aimed to assess the impact of comorbidities on prognosis in patients with three different types of COVID-19, and to provide reference for the treatment of these patients.

\section{METHODS}

\section{Study design and subjects}

This is a single-center retrospective study. A total of 1280 patients who were diagnosed with COVID-19 according to the Diagnosis and Treatment Program of Novel Coronavirus Pneumonia (Trial Seventh Edition) issued by the National Health Commission, ${ }^{15}$ and admitted to the infectious ward of Wuhan Third Hospital from January 27, 2020 to March 15, 2020, were included. There were 615 men and 665 women, aged 7-101 years, and the median age was $63(51-70)$ years old.

\section{Admission and discharge criteria}

Admission criteria were (1) having a history of epidemiology; (2) having clinical manifestations, including fever and/or respiratory symptoms; typical CT imaging features of COVID-19; normal or low total white blood cell count, or low lymphocyte count at the early stage of onset; and (3) the real-time fluorescence PCR (RT-PCR) assay was positive for SARS-CoV-2 nucleic acid. COVID-19 was diagnosed if the aforementioned criteria were met.

Criteria for discharge were (1) absence of fever for at least 3 days, (2) significant improvement of respiratory symptoms, (3) substantially improved acute exudative lesions on chest CT and (4) two throat-swab samples negative for SARS-CoV-2 obtained at least 24 hours apart. All the aforementioned four criteria need to be met for hospital discharge.

\section{Clinical typing criteria}

Clinical criteria include the following: (1) ordinary type: patients had fever and respiratory tract symptoms, and chest imaging shows pneumonia; (2) severe type: patients had any of the following items: (a) dyspnea, respiratory frequency of $\geq 30$ breaths/min; (b) blood oxygen saturation $\left(\mathrm{SpO}_{2}\right)$ of $\leq 93 \%$ at rest; (c) partial pressure of oxygen $\left(\mathrm{PaO}_{2}\right) /$ fraction of inspired oxygen $\left(\mathrm{FiO}_{2}\right)$ ratio (the ratio between $\mathrm{PaO}_{2}$ and $\left.\mathrm{FiO}_{2}\right)$ of $<300 \mathrm{~mm} \mathrm{Hg}(1 \mathrm{~mm} \mathrm{Hg}=0.133 \mathrm{kPa})$; and (3) critical type: patients had any of the following items: (a) respiratory failure requiring mechanical ventilation, (b) shock, and (c) multiple organ failure that requires ICU monitoring and treatment.

\section{Data collection}

According to the Diagnosis and Treatment Program of Novel Coronavirus Pneumonia (Trial Seventh Edition) issued by the National Health Commission, all patients were divided into ordinary, severe, and critical types based on their clinical manifestations at admission. There were 793 patients of ordinary COVID-19, 363 patients of severe COVID-19, and 124 patients of critical COVID-19. Patients' general information (age and sex), admission vital signs (body temperature, heart rate, respiratory rate, and blood pressure), clinical symptoms at admission, comorbidities, course of disease and clinical outcome were collected. Clinical outcomes include discharge, transfer and death. The condition of majority of the transferred patients was better than at admission, but they also tested positive in nucleic acid tests, so these patients were transferred to the square cabin hospital or rehabilitation station for continued treatment under the unified arrangement of Wuhan municipal headquarters for COVID-19 epidemic prevention and control; a small number of patients were transferred to other designated hospitals for continued treatment under the unified arrangement of Wuhan municipal headquarters for COVID-19 epidemic prevention and control because our hospital was no longer a designated hospital for treating COVID-19.

Comorbidities were determined based on patients' selfreport of their medical history, and also determined by various clinical tests.

\section{Statistical analysis}

Statistical analyses were performed using SPSS V.22.0. Shapiro-Wilk test was used to test the normality of continuous data; continuous data normally distributed were expressed as mean $\pm \mathrm{SD}$; continuous data non-normally distributed were presented as median (IQR). Levene's test was used to test for homogeneity of variance. Oneway analysis of variance was used to compare the mean of two samples that were normally distributed and had equal variance. Mann-Whitney $U$ test was used to compare the mean of two samples that were not normally distributed and had unequal variance. Kruskal-Wallis was used to compare multiple samples. Multivariate logistic regression and Cox proportional hazards regression models were used for multivariate survival analysis. Categorical data were 
Table 1 Comparison of clinical characteristics and outcomes in patients with three different types of COVID-19

\begin{tabular}{|c|c|c|c|c|}
\hline & $\begin{array}{l}\text { Patients with ordinary type } \\
\text { of COVID-19 }\end{array}$ & $\begin{array}{l}\text { Patients with severe type } \\
\text { of COVID-19 }\end{array}$ & $\begin{array}{l}\text { Patients with critical type of } \\
\text { COVID-19 }\end{array}$ & $P$ value \\
\hline Number & 793 & 363 & 124 & \\
\hline Age (years) & $59(49-68)$ & $67(59-74)^{*}$ & $68(60-75)^{*}$ & 0.000 \\
\hline \multicolumn{5}{|l|}{ Sex } \\
\hline Male & $354(44.6 \%)$ & $186(51.2 \%)$ & $75(60.5 \%)^{*}$ & 0.002 \\
\hline Female & $439(55.4 \%)$ & $177(48.8 \%)$ & $49(39.5 \%)^{*}$ & 0.002 \\
\hline \multicolumn{5}{|l|}{ Admission vital signs } \\
\hline Body temperature $\left({ }^{\circ} \mathrm{C}\right)$ & $36.6(36.5-37.0)$ & $36.6(36.5-37.0)$ & $37.0(36.6-37.8)^{*} \dagger$ & 0.000 \\
\hline Heart rate (beats/min) & $80(78-88)$ & $80(78-88)$ & $86(80-99)^{*} \dagger$ & 0.000 \\
\hline Respiratory rate (breaths/min) & $20(19-20)$ & $20(19-22)^{*}$ & $22(20-25)^{*}+$ & 0.000 \\
\hline Systolic blood pressure (mm Hg) & $130(120-140)$ & $130(120-140)$ & $132(122-148)^{*}$ & 0.007 \\
\hline Diastolic blood pressure $(\mathrm{mm} \mathrm{Hg})$ & $80(72-85)$ & $79(70-84)$ & $80(70-88)$ & 0.089 \\
\hline \multicolumn{5}{|l|}{ Clinical symptoms at admission } \\
\hline Fever & $542(83.5 \%)$ & $231(63.6 \%)$ & $92(74.2 \%)$ & 0.072 \\
\hline Cough & $520(65.6 \%)$ & $248(68.3 \%)$ & $95(76.6 \%)^{*}$ & 0.047 \\
\hline Sputum production & $134(16.9 \%)$ & $82(22.6 \%)$ & $35(28.2 \%)^{*}$ & 0.003 \\
\hline Fatigue & $240(30.3 \%)$ & $101(27.8 \%)$ & $37(29.8 \%)$ & 0.698 \\
\hline Chest tightness & $131(16.5 \%)$ & $114(31.4 \%)^{*}$ & $29(23.4 \%)$ & 0.000 \\
\hline Wheezing & $106(13.4 \%)$ & $113(31.1 \%)^{*}$ & $42(33.9 \%)^{*}$ & 0.000 \\
\hline Dyspnea & $39(4.9 \%)$ & $22(6.1 \%)$ & $20(16.1 \%)^{*} \dagger$ & 0.000 \\
\hline Myalgia & $42(5.3 \%)$ & $23(6.3 \%)$ & $10(8.1 \%)$ & 0.356 \\
\hline Sore throat & $64(8.1 \%)$ & $21(5.9 \%)$ & $8(6.5 \%)$ & 0.428 \\
\hline Diarrhea & $63(7.9 \%)$ & $27(7.4 \%)$ & $10(8.1 \%)$ & 0.951 \\
\hline Headache & $37(4.7 \%)$ & $11(3.0 \%)$ & $6(4.8 \%)$ & 0.411 \\
\hline \multicolumn{5}{|l|}{ Clinical outcomes } \\
\hline Discharge & $579(73.0 \%)$ & $200(55.1 \%)^{*}$ & $31(25.0 \%)^{*} \dagger$ & 0.000 \\
\hline Transfer & $211(26.6 \%)$ & $156(43.0 \%)^{*}$ & $34(27.4 \%)$ & 0.000 \\
\hline Death & $3(0.4 \%)$ & $7(1.9 \%)^{*}$ & $59(47.6 \%)^{*} \dagger$ & 0.000 \\
\hline Length of hospital stay & $15(11-18)$ & $17(15-23)^{*}$ & $16(9-26)^{*}$ & 0.000 \\
\hline Time from onset to admission & $10(7-14)$ & $10(7-14)$ & $8(7-14)$ & 0.082 \\
\hline Time from onset to discharge & $26(21-33)$ & $30(24-37)^{*}$ & $27(18-39) \dagger$ & 0.000 \\
\hline
\end{tabular}

Shapiro-Wilk test was used to test the normality of continuous data, continuous data non-normally distributed were presented as median (IQR) and compared with Kruskal-Wallis test. Categorical data were expressed by percentage and comparison was made by the $\chi^{2}$ test.

${ }^{*} \mathrm{P}<0.05$, vs ordinary patients.

$+P<0.05$, vs severe patients.

expressed by percentage, and comparison was made by the $\chi^{2}$ test and Fisher's exact test. A p value of less than 0.05 was considered as a significant difference.

\section{RESULTS}

\section{Comparison of clinical characteristics and outcomes in patients with different types of COVID-19}

As shown in table 1 , among 1280 patients with COVID-19 included in the study, 793 patients had ordinary type of COVID-19; 363 patients had severe type of COVID-19; and 124 patients had critical type of COVID-19. In terms of age, ordinary patients were relatively younger, with a median age of 59 years (IQR 49-68), and patients with severe and critical COVID-19 were relatively older, with a median age of 68 years (IQR 59-74) and 67 years (IQR 60-75), respectively. In terms of sex, critical patients had a greater proportion of men $(60.5 \%)$, followed by severe $(51.2 \%)$ and ordinary patients $(44.6 \%)$. There were statistically significant differences in the proportion of men between critical and ordinary patients $(\mathrm{p}<0.05)$. In terms of vital signs, body temperature, heart rate, respiratory rate, and systolic blood pressure in critical patients at admission were significantly higher than those of ordinary and severe patients $(\mathrm{p}<0.05)$. In terms of clinical manifestations, the common symptoms included fever, cough, sputum production, fatigue, chest tightness, wheezing, and dyspnea. Compared with ordinary patients, a significantly greater proportion of critical patients experienced cough $(76.6 \%$ vs $65.6 \%, \mathrm{p}<0.05)$, sputum production $(28.2 \%$ vs $16.9 \%, \mathrm{p}<0.05)$, wheezing $(33.9 \%$ vs $13.4 \%, \mathrm{p}<0.05)$, and dyspnea $(16.1 \%$ vs $14.9 \%$, $\mathrm{p}<0.05$ ), and a significantly greater proportion of severe patients experienced chest tightness $(31.4 \%$ vs $16.9 \%$, $\mathrm{p}<0.05)$ and wheezing $(31.1 \%$ vs $13.4 \%, \mathrm{p}<0.05)$. Critical patients had a significantly higher proportion of dyspnea compared with severe patients $(16.1 \%$ vs $6.1 \%, \mathrm{p}<0.05)$. In terms of clinical outcomes, the length of hospital stay (17 days, IQR 15-23 days; 16 days, IQR 9-26 days, vs 15 days, IQR 11-18 days; both $\mathrm{p}<0.05)$ and the time from onset to discharge (30 days, IQR 24-37 days; 27 days, IQR 18-39 days, vs 26 days, IQR $21-33$ days; both $\mathrm{p}<0.05$ ) in severe and critical patients were significantly longer than in ordinary patients. Moreover, the cure rate of critical patients 
was significantly lower than that of ordinary and severe patients $(25.0 \%$ vs $73.0 \%, 55.1 \%$; $<<0.05)$, and the cure rate of severe patients was also significantly lower than that of ordinary patients $(73.0 \%$ vs $55.1 \%, \mathrm{p}<0.05)$. In addition, the mortality rate of critical patients was significantly higher than that of ordinary and severe patients $(47.6 \%$ vs $0.4 \%, 1.9 \% ; \mathrm{p}<0.05)$, and the mortality rate of severe patients was also significantly higher than that of ordinary patients $(1.9 \%$ vs $0.4 \%, p<0.05)$. These results suggested that there were statistically significant differences between patients with different types of COVID-19 with respect to age, sex, clinical symptoms, clinical course, and clinical prognosis. Severe and critical patients were generally older; most were men and were more likely to have respiratory symptoms, especially dyspnea; and had longer treatment time, lower cure rate and higher mortality rate. In order to explore the reasons for this phenomenon and to gain a deeper understanding of the characteristics of COVID19 , we further explored whether this is associated with the presence and absence of comorbidities.

\section{Comparison of clinical characteristics and outcomes in patients with ordinary, severe, and critical COVID-19 with and without comorbidities}

Ordinary, severe, and critical patients were further divided into comorbidity and non-comorbidity groups. As shown in table 2, patients with ordinary, severe, critical COVID-19 who had comorbidities were significantly older compared with those without comorbidities (ordinary patients: 65.5, IQR 58-71 vs 54, IQR 40.5-64; severe patients: 68, IQR 61.0-74.7 vs 66, IQR 55-74; critical patients: 68, IQR $62-78$ vs 62 , IQR 52.5-73.5; both $\mathrm{p}<0.05$ ). Severe patients with comorbidities had a statistically greater proportion of men than severe patients without comorbidities $(55.7 \%$ vs $45.0 \%, \mathrm{p}<0.05)$. Ordinary, severe, and critical patients with comorbidities had significantly higher admission systolic blood pressure (ordinary patients: 135, IQR 125-147 vs 125, IQR 120-134; severe patients: 134 , IQR 124-147 vs 127, IQR 120-134; critical patients: 134, IQR $127-152$ vs 128 , IQR $119-137$; both $\mathrm{p}<0.05$ ), and a lower proportion of fever (ordinary patients: $61.0 \%$ vs $72.8 \%$; severe patients: $56.1 \%$ vs $74.2 \%$; critical patients: $69.0 \%$ vs $86.1 \%$, both $\mathrm{p}<0.05$ ) compared with ordinary, severe, and critical patients without comorbidities. Ordinary patients with comorbidities have higher proportions of wheezing $(16.3 \%$ vs $11.6 \%, \mathrm{p}<0.05)$ and dyspnea $(7.3 \%$ vs $3.4 \%, \mathrm{p}<0.05)$ compared with those without comorbidities. In terms of clinical outcomes, no significant differences were found in cure rate, transfer-out rate, and mortality rate between ordinary patients with and without comorbidities, but the length of hospital stay (15, IQR $11.3-19)$ vs 15 , IQR 11-18), $\mathrm{p}<0.05)$, and time from onset to discharge (28(IQR,22-36) vs $25(\mathrm{IQR}, 20-31), \mathrm{p}<0.05)$ were significantly longer in ordinary patients with comorbidities than those in ordinary patients who did not had comorbidities. Severe patients with comorbidities had significantly higher cure rate $(63.7 \%$ vs $43.0 \%, \mathrm{p}<0.05)$, and significantly lower transfer-out rate $(34.0 \%$ vs $56.2 \%, \mathrm{p}<0.05)$ than those without comorbidities. The mortality rate of severe patients with comorbidities was higher than that of patients without comorbidities, but this difference was not statistically significant $(2.4 \%$ vs $41.3 \%, \mathrm{p}>0.05)$. There was no significant difference in the length of hospital stay and the time from onset to discharge between severe patients with and without comorbidities $(p>0.05)$. No statistically significant difference was found in the length of hospital stay and the time from onset to discharge between critical patients with and without comorbidities. Critical patients with comorbidities had significantly lower cure rate $(19.3 \%$ vs $38.9 \%, \mathrm{p}<0.05)$ and significantly higher mortality rate $(53.4 \%$ vs $33.3 \%, \mathrm{p}<0.05)$ compared with those without comorbidities.

These results indicated that comorbidities can significantly affect the clinical course (length of hospital stay, time from onset to discharge) of ordinary patients, reduce the cure rate, and increase mortality rate of critical patients. In order to explore the reasons for this phenomenon, we further analyzed which comorbidities can cause this phenomenon.

\section{Distribution of comorbidities in patients with different types of COVID-19}

As shown in table 3, among 1280 patients included in the study, 600 (46.9\%) patients had at least one comorbidity; comorbidities that had a a frequency of greater than $1 \%$ included hypertension $(n=423,33.0 \%)$, diabetes $(n=184$, $14.4 \%)$, coronary heart disease $(n=102,8.0 \%)$, COPD $(\mathrm{n}=30,2.3 \%)$, arrhythmia $(\mathrm{n}=28,2.2 \%)$, chronic renal failure $(n=20,1.6 \%)$, thyroid disease $(n=20,1.6 \%)$ and malignant tumors $(n=13,1.0 \%)$. Critical patients had statistically higher proportions of hypertension $(46.8 \%$ vs $26.1 \%, \mathrm{p}<0.05)$, arrhythmia ( $4.8 \%$ vs $1.1 \%, \mathrm{p}<0.05)$, and chronic renal failure $(3.2 \%$ vs $0.5 \%, \mathrm{p}<0.05)$ compared with ordinary patients. Severe patients had statistically higher proportions of hypertension $(43.5 \%$ vs $26.1 \%$, $\mathrm{p}<0.05)$, diabetes $(19.8 \%$ vs $11.5 \%, \mathrm{p}<0.05)$, coronary heart disease $(11.0 \%$ vs $6.1 \%, \mathrm{p}<0.05)$, arrhythmia $(3.6 \%$ vs $1.1 \%, \mathrm{p}<0.05)$, and chronic renal failure $(3.3 \%$ vs $0.5 \%$, $\mathrm{p}<0.05)$ compared with ordinary patients.

We further analyzed the number and proportion of patients who had one and two or more comorbidities; the results showed that $385(64.2 \%)$ patients had only one comorbidity, including 195 (50.6\%) ordinary patients, 130 $(33.8 \%)$ severe patients, and $60(15.6 \%)$ critical patients; 215 (35.8\%) patients had $\geq 2$ comorbidities, including 105 $(48.8 \%)$ ordinary patients, $82(38.1 \%)$ severe patients, and $28(13.8 \%)$ critical patients. No significant difference in prognosis was found between ordinary, severe, and critical patients with one comorbidity and those with $\geq 2$ comorbidities $(p>0.05)$, indicating that the number of comorbidities did not directly affect the clinical prognosis in patients with different types of COVID-19. Then we selected top six most common comorbidities, hypertension, diabetes, coronary heart disease, COPD, arrhythmia, and chronic renal failure to further investigate which comorbidities can impact prognosis in patients with COVID-19.

\section{Relationship between each comorbidity and clinical characteristics, course, and outcomes in patients with different types of COVID-19}

As shown in table 4, ordinary patients with hypertension, diabetes, coronary heart disease, and COPD were 


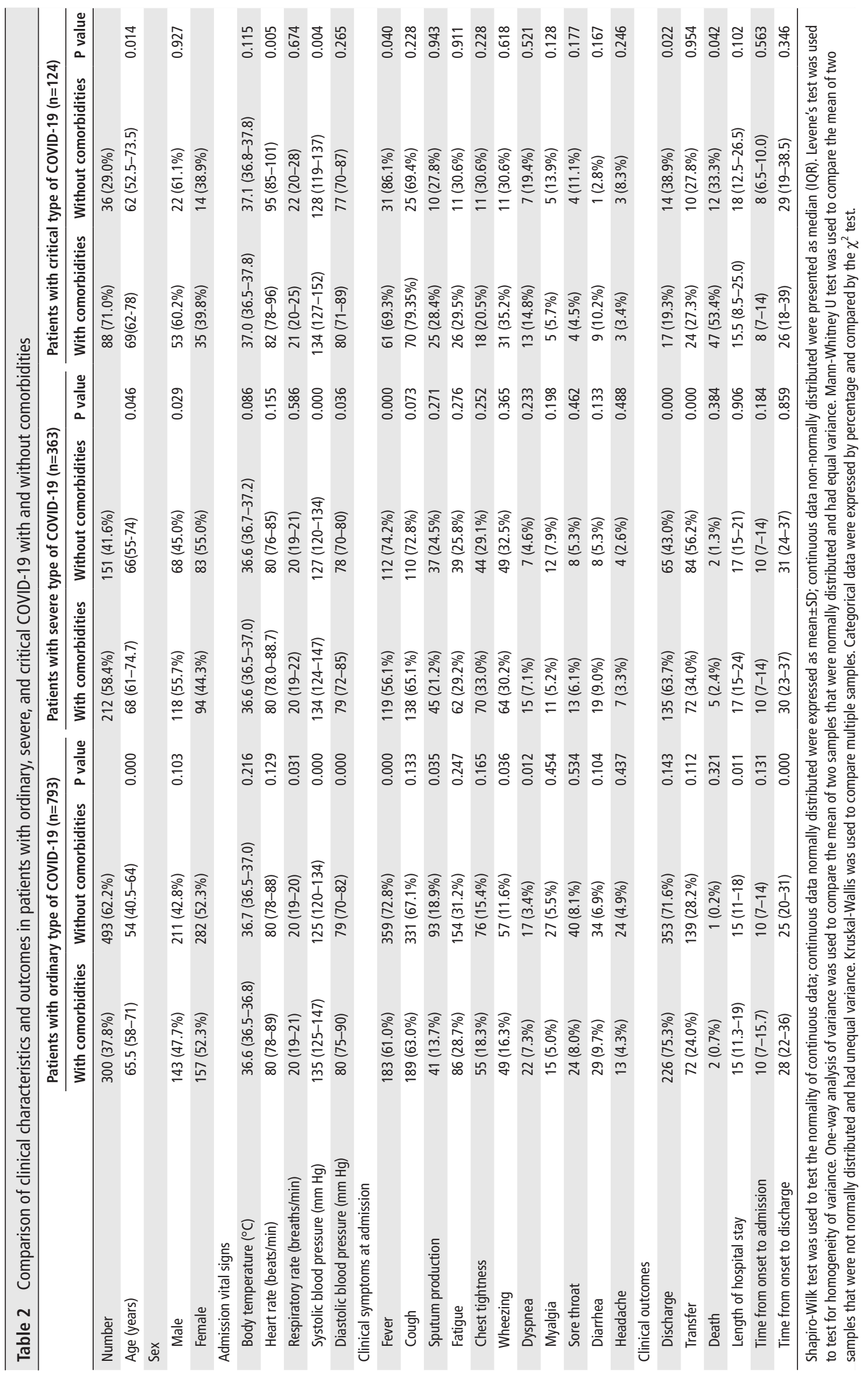




\begin{tabular}{|c|c|c|c|c|c|}
\hline & Total $(n=1280)$ & $\begin{array}{l}\text { Patients with ordinary type of } \\
\text { COVID-19 }(n=793)\end{array}$ & $\begin{array}{l}\text { Patients with severe type of } \\
\text { COVID-19 }(n=363)\end{array}$ & $\begin{array}{l}\text { Patients with critical type of } \\
\text { COVID-19 }(n=124)\end{array}$ & $P$ value \\
\hline \multicolumn{6}{|l|}{ Comorbidities } \\
\hline Hypertension & $423(33.0 \%)$ & $207(26.1 \%)$ & $158(43.5 \%)^{*}$ & $58(46.8 \%)^{*}$ & 0.000 \\
\hline Diabetes & $184(14.4 \%)$ & $91(11.5 \%)$ & $72(19.8 \%)^{*}$ & $21(16.9 \%)$ & 0.001 \\
\hline Coronary heart disease & $102(8.0 \%)$ & $48(6.1 \%)$ & $40(11.0 \%)^{*}$ & $14(11.3 \%)$ & 0.005 \\
\hline $\begin{array}{l}\text { Chronic obstructive pulmonary } \\
\text { disease }\end{array}$ & $30(2.3 \%)$ & $15(1.9 \%)$ & $10(2.8 \%)$ & $5(4.0 \%)$ & 0.270 \\
\hline Arrhythmia & $28(2.2 \%)$ & $9(1.1 \%)$ & $13(3.6 \%)^{*}$ & $6(4.8 \%)^{*}$ & 0.006 \\
\hline Chronic renal failure & $20(1.6 \%)$ & $4(0.5 \%)$ & $12(3.3 \%)^{*}$ & $4(3.2 \%)^{*}$ & 0.001 \\
\hline Malignant tumors & $13(1.0 \%)$ & $9(1.1 \%)$ & 0 & $4(3.2 \%)$ & 0.280 \\
\hline Cirrhosis & $8(0.6 \%)$ & $2(0.3 \%)$ & $3(0.8 \%)$ & $3(2.4 \%)^{*}$ & 0.011 \\
\hline Chronic hepatitis B & $9(0.7 \%)$ & $7(0.9 \%)$ & 0 & $2(1.6 \%)$ & 0.257 \\
\hline Thyroid disease & $20(1.6 \%)$ & $15(1.9 \%)$ & $4(1.1 \%)$ & $1(0.8 \%)$ & 0.151 \\
\hline Cerebrovascular disease & $13(1.0 \%)$ & $6(0.8 \%)$ & $5(1.4 \%)$ & $2(1.6 \%)$ & 0.168 \\
\hline Bronchiectasis & $6(0.5 \%)$ & $6(0.8 \%)$ & 0 & 0 & \\
\hline Allergic asthma & $6(0.5 \%)$ & $1(0.1 \%)$ & $4(1.1 \%)$ & $1(0.8 \%)$ & 0.062 \\
\hline \multicolumn{6}{|l|}{ Number of comorbidities } \\
\hline One comorbidity & $385(64.2 \%)$ & $195(50.6 \%)$ & $130(33.8 \%)^{*}$ & $60(15.6 \%)^{*} \dagger$ & 0.000 \\
\hline$\geq 2$ comorbidities & $215(35.8 \%)$ & $105(48.8 \%)$ & $82(38.1 \%)$ & $28(13.0 \%)^{*} \dagger$ & 0.000 \\
\hline
\end{tabular}

Categorical data were expressed by percentage and compared by the $\chi^{2}$ test.

${ }^{*} \mathrm{P}<0.05$, vs ordinary patients.

$+P<0.05$, vs severe patients.

significantly older than those without comorbidities $(\mathrm{p}<0.05)$, but no significant difference was found in the proportions of men and women between ordinary patients with and without those comorbidities $(p>0.05)$. In terms of clinical symptoms, ordinary patients without hypertension, diabetes, and coronary heart disease had a significantly higher proportion of fever compared with patients who had those comorbidities $(\mathrm{p}<0.05)$. Ordinary patients without diabetes had significantly higher proportions of cough and sputum production than those had diabetes $(\mathrm{p}<0.05)$. Ordinary patients with hypertension had significantly higher proportions of wheezing and dyspnea, and lower proportion of headaches compared with those who had hypertension $(\mathrm{p}<0.05)$. Time from onset to discharge was significantly longer in ordinary patients with hypertension compared with those without hypertension (27, IQR $22-35$ vs 25 , IQR 21-32; p $<0.05$ ).

As shown in table 5 , severe patients with hypertension and coronary heart disease were significantly older than patients without those comorbidities. In terms of clinical symptoms, severe patients without hypertension, diabetes had a higher proportion of fever compared with those with hypertension and diabetes, while no significant difference was found in other symptoms. In terms of clinical outcomes, the cure rate $(67.1 \%$ vs $45.9 \%, \mathrm{p}<0.05)$ was significantly higher, and the transfer-out rate $(29.7 \%$ vs $53.1 \%, \mathrm{p}<0.05)$ was significantly lower in severe patients with hypertension than in those without hypertension $(p<0.05)$. Although the length of hospital stay, time from onset to discharge, and time to cure were longer in severe patients with hypertension than in those without hypertension, but the differences were not statistically significant.

As shown in table 6, critical patients with hypertension were significantly older than those without hypertension, and no significant difference in gender and clinical symptoms was found. In terms of clinical outcomes, critical patients with diabetes have a significantly lower cure rate $(14.3 \%$ vs $27.2 \%)$ and a significantly high mortality rate (71.4\% vs $42.7 \%)$ compared with those without diabetes.

For critical patients, results from multivariate logistic regression model containing age, gender, and comorbidities showed that male patients had a significantly increased risk of death than female patients (OR=4.395, 95\% CI 1.896 to 10.185, $\mathrm{p}<0.05)$; patients with diabetes were at increased risk of death $(\mathrm{OR}=3.542,95 \% \mathrm{CI} 1.167$ to 10.750 , $\mathrm{p}<0.05$; online supplemental table S1). Results from Cox proportional hazards regression model containing age, gender, and comorbidities also showed that diabetes was significantly associated with increased risk of death (HR $2.03,95 \%$ CI 1.127 to $3.662 ; \mathrm{p}<0.05)$, and men had a higher risk of death than women (HR 2.91, 95\% CI 1.592 to $5.336, \mathrm{p}<0.05$; online supplemental table S2) (online supplemental figure S1).

\section{DISCUSSION}

Since COVID-19 emerged gradually in December 2019, many researchers have reported the epidemiology, clinical manifestations, laboratory, imaging finding, and clinical prognosis of COVID-19, as well as associated risk factors. Wang et al found that the presence of comorbidities is an independent risk factor for severity and even death in patients with COVID-19. ${ }^{10}$ The impact of comorbidities on patients with mild COVID-19 is still unclear. In this study, according to the clinical manifestations of patients at admission, all patients were divided into ordinary, severe, and critical types, the impact of comorbidities on prognosis in patients with three different types of COVID-19 were assessed.

In this study, comparison of the clinical data of patients with different types of COVID-19 showed that compared with ordinary patients, severe and critical patients were 


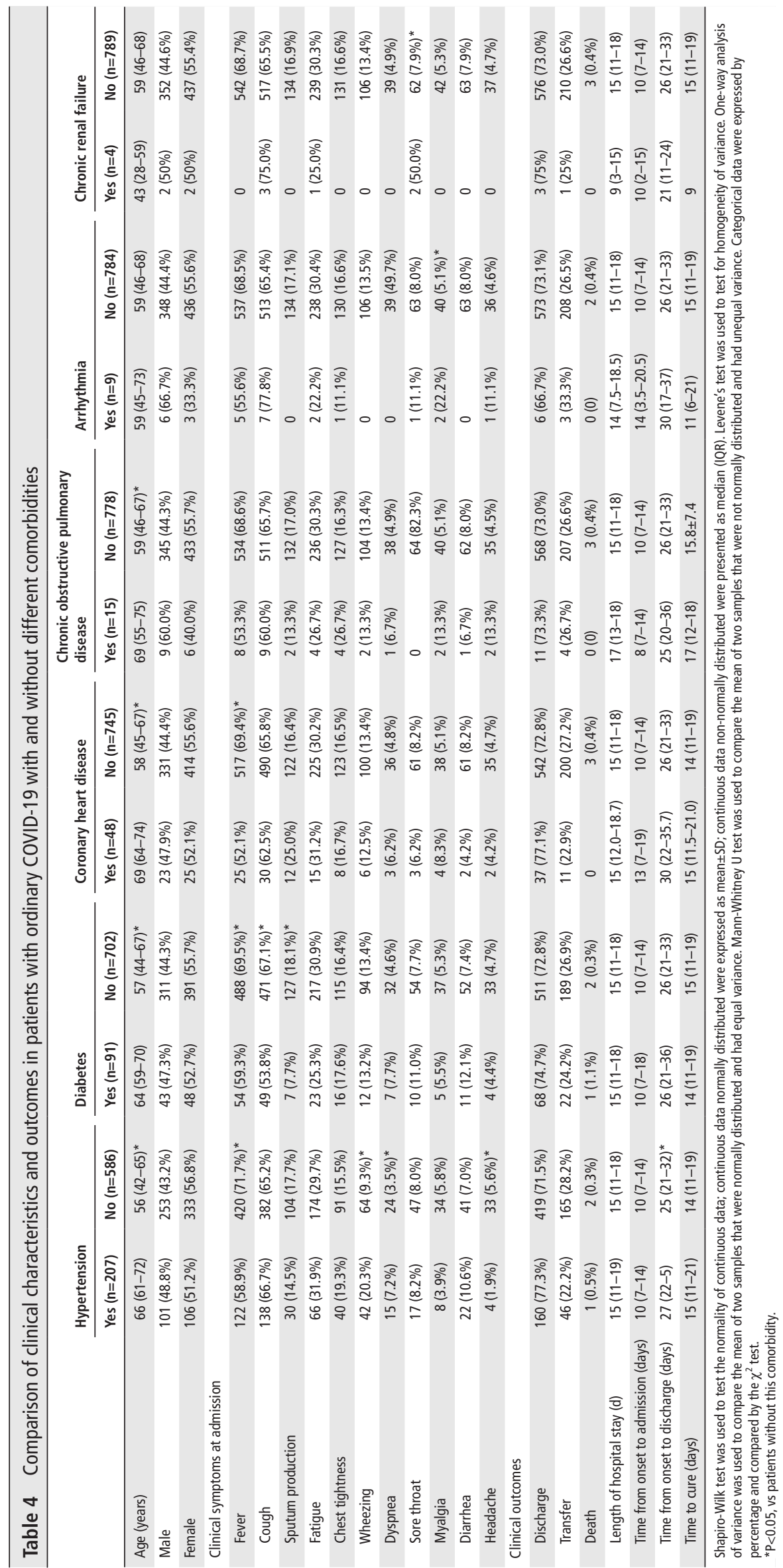




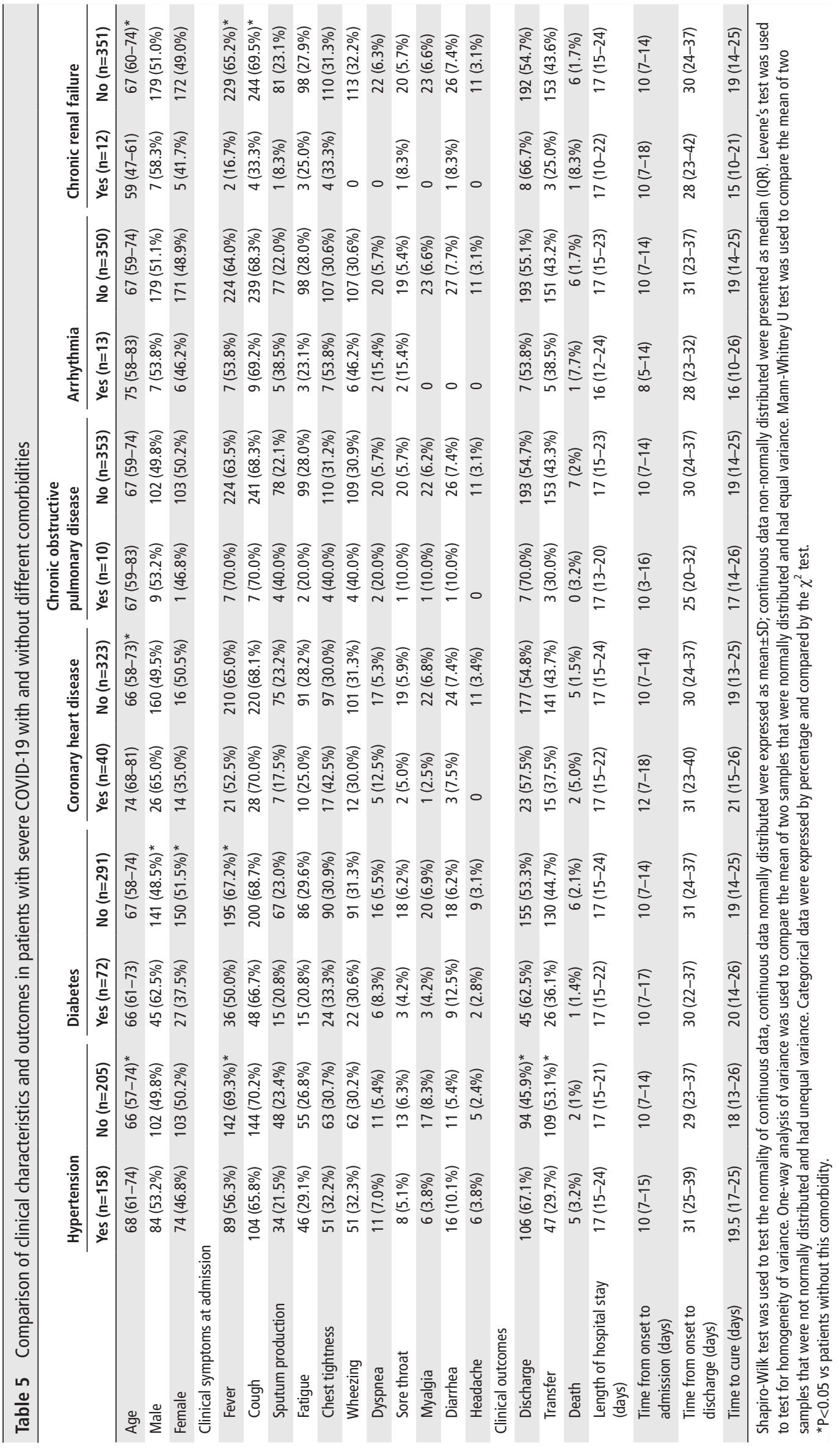




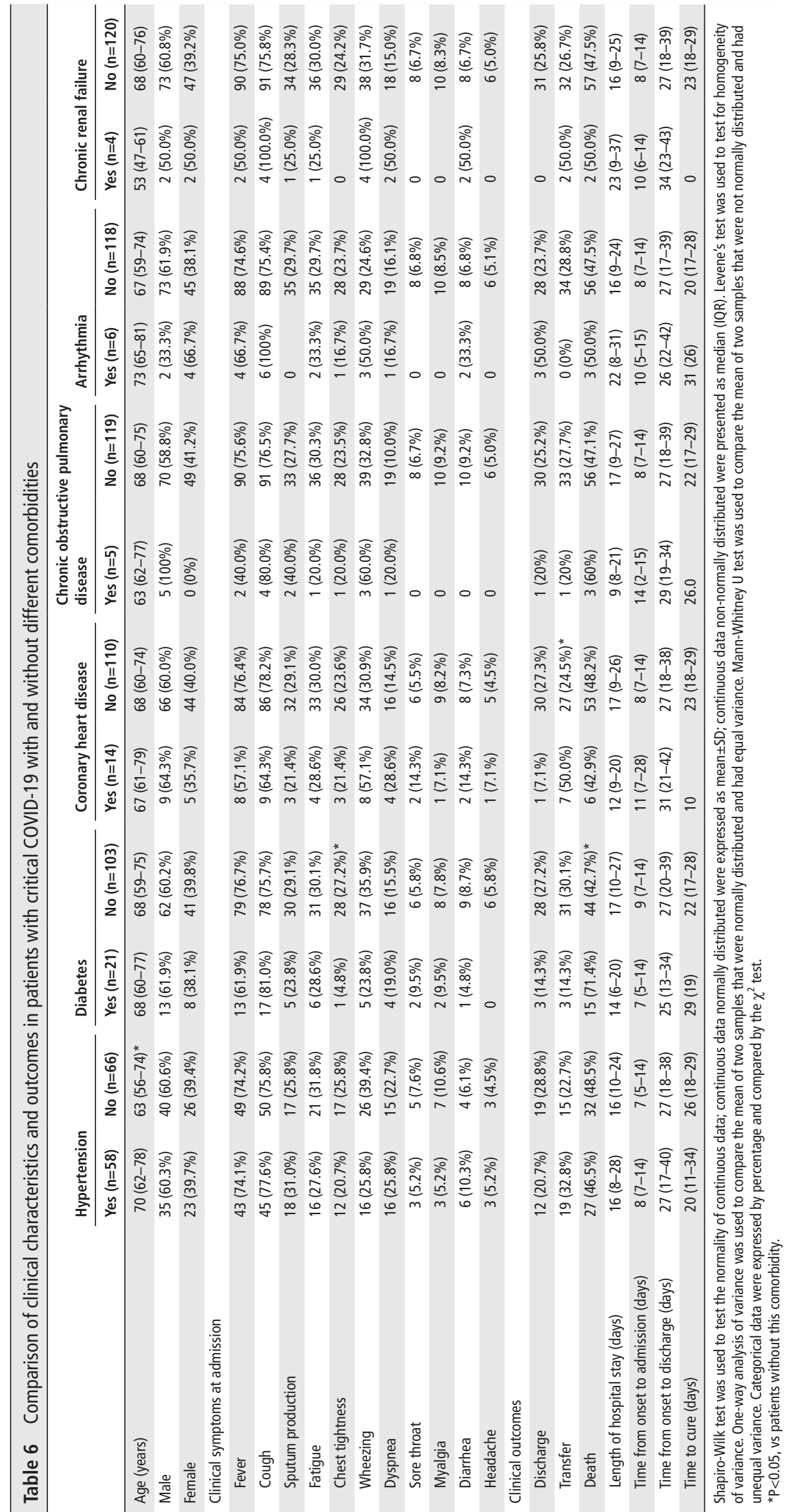


significantly older; had higher proportion of men, higher proportions of severe symptoms such as cough, sputum production, chest tightness, wheezing, and dyspnea; lower cure rate; higher mortality rate; and longer hospital stay. These results are consistent with the finding from previous studies. ${ }^{10}{ }^{16}$ Unlike the previous studies, the present study included a large sample size and explored the clinical outcomes in all patients, including cure rate, transfer out, and death, which can truly reflect the clinical course and outcomes in patients with different types of COVID-19.

Then we further analyzed the impact of the presence or absence of comorbidities on the clinical outcomes of patients with different types of COVID-19, the results showed that for ordinary patients, there was no difference in the cure rates and mortality rates between patients with or without comorbidities, but the length of hospital stay and time to cure in ordinary patients with comorbidities were obviously longer compared with those without comorbidities, indicating that comorbidities cannot affect the cure rate and mortality rate of ordinary patients but can prolong patients' clinical course. For severe patients, the cure rate was higher, and the transfer-out rate was lower in patients with comorbidities compared with those without comorbidities; the low cure rate of patients without comorbidities may be related to the high transfer-out rate. Moreover, the mortality rate in severe patients with comorbidities were higher than those without comorbidities, but this difference was not statistically significant, this may be associated with a small number of deaths; and there is no significant difference in clinical course between severe patients with or without comorbidities. For critical patients, the cure rate was low and the mortality rate was high in patients with comorbidities compared those without comorbidities, indicating that comorbidities can increase the mortality and reduce the cure rate in ptients with critical COVID-19.

We found that the most common comorbidities in patients with different types of COVID-19 were hypertension, diabetes, coronary heart disease, COPD, arrhythmia, chronic renal failure, malignant tumors, cirrhosis, chronic hepatitis B, thyroid disease, and cerebrovascular disease, which are consistent with the comorbidities reported in the literature. ${ }^{14}$ Compared with ordinary patients, critical patients had higher proportions of hypertension, arrhythmia, and chronic renal failure, severe patients with COVID-19 had higher proportions of hypertension, diabetes, coronary heart disease, arrhythmia, and chronic renal failure. In addition, we found that some patients have multiple comorbidities, a significantly higher proportion of severe and critical patients had two or more comorbidities compared with patients with ordinary COVID-19. Guan et $a l^{14}$ also showed that the number of patients with severe and critical COVID-19 having one or more comorbidities was increased, and patients with $\geq 2$ comorbidities were older and had more obvious clinical manifestations than patients with one comorbidity, but in this study, no significant difference was found in the clinical data between patients with $\geq 2$ comorbidities and those with one comorbidity.

Furthermore, we selected top six most prevalent comorbidities as variables and explored their impact on patients with three different types of COVID-19. For ordinary patients, those with hypertension, diabetes, coronary heart disease, and COPD were significantly older than patients without comorbidities; no significant difference was found in sex between ordinary patients with and without these comorbidities. Time from onset to discharge, length of hospital stay and time to cure were both significantly prolonged in ordinary patients with hypertension compared those without hypertension, indicating that hypertension can affect the disease course of ordinary patients. For severe patients, those with hypertension and coronary heart disease were significantly older than patients without comorbidities; in terms of clinical outcomes, the cure rate of severe patients with hypertension was higher than those without hypertension; this may be due to the high transfer-out rate of patients without hypertension; time from onset to discharge, length of hospital stay and time to cure were both longer in severe patients with hypertension than those without hypertension, but the differences were not statistically significant. For critical patients, those with hypertension are significantly older, and there were no significant differences in sex and clinical symptoms between patients with and without hypertension; in terms of clinical outcomes, critical patients with diabetes had lower cure rate, higher mortality rate, prolonged time to cure, and higher risk of death, indicating that diabetes may be a risk factor for prognosis in patients with critical COVID-19. A previous study showed that diabetes was considered to be an independent risk factor for severe complications and death from SARS-CoV-1) during the 2002-2003 outbreak. ${ }^{17}$ Similarly, the presence of diabetes was associated with three times higher risk for hospitalization, and four times higher risk for ICU admission among patients with influenza A in 2009. ${ }^{18}$ During the outbreak of the Middle East Respiratory Syndrome-Coronavirus in 2012, the prevalence of diabetes was nearly $50 \%$, and the mortality rate of patients with diabetes was as high as $35 \% .{ }^{19} 20$ A recent study showed that a higher proportion of patients with COVID-19 had diabetes, with a high mortality rate of $34 \% .{ }^{21}$ The findings of the aforementioned studies have shown that diabetes plays an important role in the development of acute respiratory disease, including COVID-19, and this may be due to the reason that immune system function is suppressed in diabetic patients.

To our knowledge, our study is the first to investigate the impact of comorbidities on clinical prognosis in patients with different types of COVID-19, and provide reference for treatment of these patients. However, this study has some limitations, first, this is a single-center retrospective study, which may introduce selection bias; second, some patients included in this study did not test negative and transferred to other medical institutions to continue their treatment, so the clinical outcomes can not be fully reflected.

In summary, our findings suggested that (1) compared with ordinary patients, severe and critical patients were older, had higher proportion of men, higher proportions of severe symptoms such as cough, sputum production, chest tightness, wheezing, and dyspnea, lower cure rate, higher mortality rate, longer hospital stay and time to cure; (2) comorbidities did not affect the cure rate and mortality rate of ordinary patients but can prolong patients' clinical course, including length of hospital stay and time to cure; comorbidities have no obvious impact on the cure rate, mortality rate and clinical course of severe patients; comorbidities increase the mortality rate, reduce the cure 
rate, and prolong the clinical course of critical patients; (3) hypertension may be an important factor affecting the clinical course of ordinary patients, and diabetes may be an important factor affecting the clinical course and prognosis of critical patients.

Acknowledgements We thank all subjects enrolled in the present study.

Contributors $\mathrm{ZL}$ and PL designed the study and revised the manuscript. $\mathrm{JH}$ and $\mathrm{HF}, \mathrm{QL}, \mathrm{MX}, \mathrm{DX}$ and $\mathrm{JH}$ collated the data and carried out the data analyses. HF wrote the main manuscript text. All authors have read and approved the final submitted manuscript.

Funding This work was supported by the Health Family Planning Research Fund of Wuhan City (grant number WX18M01), the Wuhan City 'Huanghe Talent' Program, and the Wuhan Application Foundation Frontier Project (grant number 2019020701011471).

Competing interests None declared.

Patient consent for publication Not required.

Ethics approval This study is approved by the ethics committee of Wuhan Third Hospital, Tongren Hospital of Wuhan University (KY2020-014). Informed consent was waived due to the retrospective nature of this study.

Provenance and peer review Not commissioned; externally peer reviewed.

Data availability statement All data relevant to the study are included in the article or uploaded as supplementary information.

Supplemental material This content has been supplied by the author(s). It has not been vetted by BMJ Publishing Group Limited (BMJ) and may not have been peer-reviewed. Any opinions or recommendations discussed are solely those of the author(s) and are not endorsed by BMJ. BMJ disclaims all liability and responsibility arising from any reliance placed on the content. Where the content includes any translated material, BMJ does not warrant the accuracy and reliability of the translations (including but not limited to local regulations, clinical guidelines, terminology, drug names and drug dosages), and is not responsible for any error and/or omissions arising from translation and adaptation or otherwise.

Open access This is an open access article distributed in accordance with the Creative Commons Attribution Non Commercial (CC BY-NC 4.0) license, which permits others to distribute, remix, adapt, build upon this work noncommercially, and license their derivative works on different terms, provided the original work is properly cited, an indication of whether changes were made, and the use is non-commercial. See: http://creativecommons.org/ licenses/by-nc/4.0/.

ORCID iD

Zhanghua Li http://orcid.org/0000-0002-9919-8111

\section{REFERENCES}

1 Huang C, Wang Y, Li X, et al. Clinical features of patients infected with 2019 novel coronavirus in Wuhan, China. Lancet 2020;395:497-506.
2 Chen N, Zhou M, Dong X, et al. Epidemiological and clinical characteristics of 99 cases of 2019 novel coronavirus pneumonia in Wuhan, China: a descriptive study. Lancet 2020;395:507-13.

3 International Committee on Taxonomy of Viruses. Naming the 2019 coronavirus, 2020. Available: https://talk.ictvonline.org/

4 Notice of the National Health Commission of the People's Republic of China on revising the english name of novel coronavirus pneumonia, 2020. Available: http://www.nhc.gov.cn/yzygj/s7653p/202002/33393aa53d984ccdb1053a52 b6bef810.shtml [Accessed 29 Feb 2020].

5 Li Q, Guan X, Wu P, et al. Early transmission dynamics in Wuhan, China, of novel coronavirus-infected pneumonia. N Engl J Med 2020;382:1199-207.

6 Chan JF-W, Yuan S, Kok K-H, et al. A familial cluster of pneumonia associated with the 2019 novel coronavirus indicating person-to-person transmission: a study of a family cluster. Lancet 2020;395:514-23.

7 World Health Organisation. Coronavirus disease (COVID-19) pandemic. Available: https://www.who.int [Accessed 10 Mar 2020].

8 Guan W-J, Ni Z-Y, Hu Y, et al. Clinical characteristics of coronavirus disease 2019 in China. N Engl J Med 2020;382:1708-20.

9 Li L-Q, Huang T, Wang Y-Q, et al. COVID-19 patients' clinical characteristics, discharge rate, and fatality rate of meta-analysis. J Med Virol 2020;92:577-83.

10 Wang D, Yin Y, Hu C, et al. Clinical course and outcome of 107 patients infected with the novel coronavirus, SARS-CoV-2, discharged from two hospitals in Wuhan, China. Crit Care 2020;24:188.

11 Zhang J, Yu M, Tong S, et al. Predictive factors for disease progression in hospitalized patients with coronavirus disease 2019 in Wuhan, China. J Clin Virol 2020;127:104392.

12 Zhou F, Yu T, Du R, et al. Clinical course and risk factors for mortality of adult inpatients with COVID-19 in Wuhan, China: a retrospective cohort study. Lancet 2020;395:1054-62.

13 Yang J, Zheng Y, Gou X, et al. Prevalence of comorbidities and its effects in patients infected with SARS-CoV-2: a systematic review and meta-analysis. Int $J$ Infect Dis 2020;94:91-5.

14 Guan W-J, Liang W-H, Zhao Y, et al. Comorbidity and its impact on 1590 patients with COVID-19 in China: a nationwide analysis. Eur Respir J 2020;55:2000547

15 National Health Commission. Diagnosis and treatment program of novel coronavirus pneumonia (trial seventh edition). Available: http://www.nhc.gov. cn/yzygj/s7652m/202003/a31191442e29474b98bfed5579d5af95.shtml

16 Feng Y, Ling Y, Bai T, et al. COVID-19 with different severities: a multicenter study of clinical features. Am J Respir Crit Care Med 2020;201:1380-8.

17 Yang JK, Feng Y, Yuan MY, et al. Plasma glucose levels and diabetes are independent predictors for mortality and morbidity in patients with SARS. Diabet Med 2006;23:623-8.

18 Allard $\mathrm{R}$, Leclerc $\mathrm{P}$, Tremblay $\mathrm{C}$, et al. Diabetes and the severity of pandemic influenza A (H1N1) infection. Diabetes Care 2010;33:1491-3.

19 Badawi A, Ryoo SG. Prevalence of comorbidities in the middle East respiratory syndrome coronavirus (MERS-CoV): a systematic review and meta-analysis. Int $J$ Infect Dis 2016:49:129-33.

20 Alraddadi BM, Watson JT, Almarashi A, et al. Risk factors for primary middle East respiratory syndrome coronavirus illness in humans, Saudi Arabia, 2014. Emerg Infect Dis 2016;22:49-55.

21 Bhatraju PK, Ghassemieh BJ, Nichols M, et al. Covid-19 in Critically III Patients in the Seattle Region - Case Series. N Eng/ J Med 2020;382:2012-22. 